\title{
Tiaochang Xiaoliu Decoction Granules prevent the recurrence of colorectal adenoma: a study protocol for a randomized controlled trial
}

\author{
Cailing Zhong ${ }^{1 \#}$, Shaohua Wu ${ }^{2 \#}$, Chunhui Chen ${ }^{1}$, Haocheng Huang ${ }^{2}$, Jiahao $\mathrm{Mo}^{2}$, Xiaobo Yang', \\ Yan Chen ${ }^{1}$, Beiping Zhang ${ }^{1}$ \\ ${ }^{1}$ Department of Gastroenterology, The Second Affiliated Hospital of Guangzhou University of Traditional Chinese Medicine, Guangzhou, China; \\ ${ }^{2}$ Guangzhou University of Traditional Chinese Medicine, Guangzhou, China \\ \#These authors contributed equally to this work. \\ Correspondence to: Yan Chen; Beiping Zhang. Department of Gastroenterology, The Second Affiliated Hospital of Guangzhou University of \\ Traditional Chinese Medicine, 111 Dade Road, Yuexiu District, Guangzhou 510120, China. Email: 13535071973@163.com; doctorzbp@163.com.
}

\begin{abstract}
Background: With the changes in lifestyle and diet, the incidence and mortality of colorectal cancer (CRC) is increasing in China. CRC mainly develops from colorectal adenomas (CRAs). There is a lack of chemopreventative drugs with definite efficacy for CRAs. Tiaochang Xiaoliu Decoction (TXD) was developed by Professor Yunjian Luo and has been used clinically over the last ten years for the prevention of CRA recurrence. To facilitate its clinical use, TXD was further standardized and produced as "Tiaochang Xiaoliu Decoction Granules (TXDG)". A study was designed to investigate the preventive effects of TXDG on the recurrence of CRA.

Methods: A randomized, double-blinded, controlled, and multi-center experiment is proposed to assess the effectiveness and safety of TXDG. Patients with CRAs (after complete polypectomy under colonoscopy) will be randomly divided into two groups, one will be treated with TXDG (the TXDG group) and the other will be treated with a TXDG mimetic agent (the TXDG mimetic group). The patients will be treated for 6 months and followed up for 3 years. Follow-up colonoscopy is expected to be carried out within 1 to 3 years after the baseline examinations. The primary outcome measure is adenoma detection rate within 1 to 3 years. The secondary outcome measures are the number, location, and pathology of the adenomas, and the polyp detection rate.
\end{abstract}

Discussion: Reliable objective evidence will be provided to evaluate the efficacy and safety of TXDG as an accessorial therapy for CRA occurrence in post-polypectomy patients.

Trial registration: ChiCTR2000035257.

Keywords: Traditional Chinese medicine; colorectal adenoma (CRA); randomized controlled trial; Tiaochang Xiaoliu Decoction Granules (TXDG)

Submitted Jan 14, 2021. Accepted for publication Apr 12, 2021.

doi: 10.21037/apm-21-650

View this article at: http://dx.doi.org/10.21037/apm-21-650

\section{Introduction}

Colorectal cancer (CRC) is the third most common malignant tumor and represents a considerable challenge to public health worldwide (1). Efforts are urgently needed to reduce the morbidity and mortality of CRC patients. Most CRCs develop from adenomas through the adenoma- carcinoma sequence which may take more than 10 years (2). Endoscopic resection of CRAs is the first line of therapy for the prevention of CRC. Endoscopic adenoma removal can prevent $75 \%$ of CRCs. However, CRAs have a high recurrence rate after endoscopic treatment, and related studies have shown that the recurrence rate of CRA is as 
Table 1 Standard formulation of TXDG

\begin{tabular}{llc}
\hline English name & Latin name & Dose $(\mathrm{g})$ \\
\hline Codonopsis radix & Codonopsis pilosula (Franch.) Nannf. & 15 \\
Atractylodis macrocephalae rhizoma & Atractylodes macrocephala Koidz & 15 \\
Astragali radix & Astragalus memeranaceus (Fisch.) Bge & 15 \\
Coicis semen & Coix lacryma-jobi L.var.mayuen (Roman.) Stapf & 30 \\
Amomi fructus rotundus & Amomum krauanh pierreex gagnep. & 10 \\
Hedyotidis diffusae herba & Hedyotis diffusa Willd. & 30 \\
Curcumae rhizoma & Curcuma phaeocaulis Val. & 15 \\
Notoginseng radix et rhizoma & Panax notoginseng (Burk.) F.H.Chen & 10 \\
Glycyrrhizae radix et rhizoma & Glycyrrhiza uralensis Fisch. & 5 \\
\hline
\end{tabular}

high as $37 \% 1$ year after surgery (3).

The secondary method for the prevention of CRAs requires comprehensive treatments. However, current chemopreventative methods used in Western medicine may have severe side effects and uncertain efficacy. While traditional Chinese medicine (TCM) has definite advantages in the secondary prevention of CRAs, the methods can be too general and vague and require further optimization. Additionally, there is a paucity of high-quality and highlevel evidence-based research examining the efficacy of TCM in the prevention of CRAs.

Tiaochang Xiaoliu Decoction Granules (TXDG) were developed by Professor Yunjian Luo who has made great contributions to the prevention of CRA recurrence postpolypectomy over the last ten years. TXDG is a TCM empirical formula and the composition is listed in Table 1 . Previous studies have shown that the recurrence rate of CRAs in patients treated with Tiaochang Xiaoliu Decoction (TXD)-based decoction was $12.5 \%$, compared with $30.6 \%$ in the control group. However, further large scale clinical studies are essential to fully evaluate the efficacy. Therefore, a randomized, double-blinded, controlled, and multicenter trial is proposed to investigate the effects of TXDG on the recurrence of CRAs in post-polypectomy patients. We present the following article in accordance with the SPIRIT reporting checklist (available at http://dx.doi.org/10.21037/ apm-21-650).

\section{Methods}

\section{Study design}

This multicenter, randomized, controlled, and doubleblinded trial is registered with the Chinese Clinical Trial
Registry (ChiCTR2000035257). Professional researchers will introduce the study to patients, offering them relevant information and obtaining informed consent. All patients must provide written informed consent prior to registration. The flowchart of the study is shown in Figure 1.

\section{Ethics}

The trial complies with the Good Clinical Practice Guidelines and the Declaration of Helsinki (as revised in 2013) (4). Ethics approval of this trial has been obtained from the ethic committee of the Guangdong Provincial Hospital of Traditional Chinese Medicine (No. BF2020103-01). All subjects will be requested to sign written informed consent forms.

\section{Participants}

A total of 260 eligible Chinese patients who satisfy the inclusion criteria will be recruited from five hospitals in China through posters advertised at the hospitals. A total of 60 patients will be recruited from the Guangdong Provincial Hospital of Traditional Chinese Medicine, 50 patients from The First Affiliated Hospital of Naval Medical University, 50 patients from the Wangjing Hospital Affiliated to the Chinese Academy of Traditional Chinese Medicine, 50 patients from the Southern Hospital of Southern Medical University, and 50 patients from the Shenzhen Traditional Chinese Medical Hospital.

\section{Sample size}

Our previous study (5) demonstrated that the CRA recurrence rate in patients in the first year after TXD 


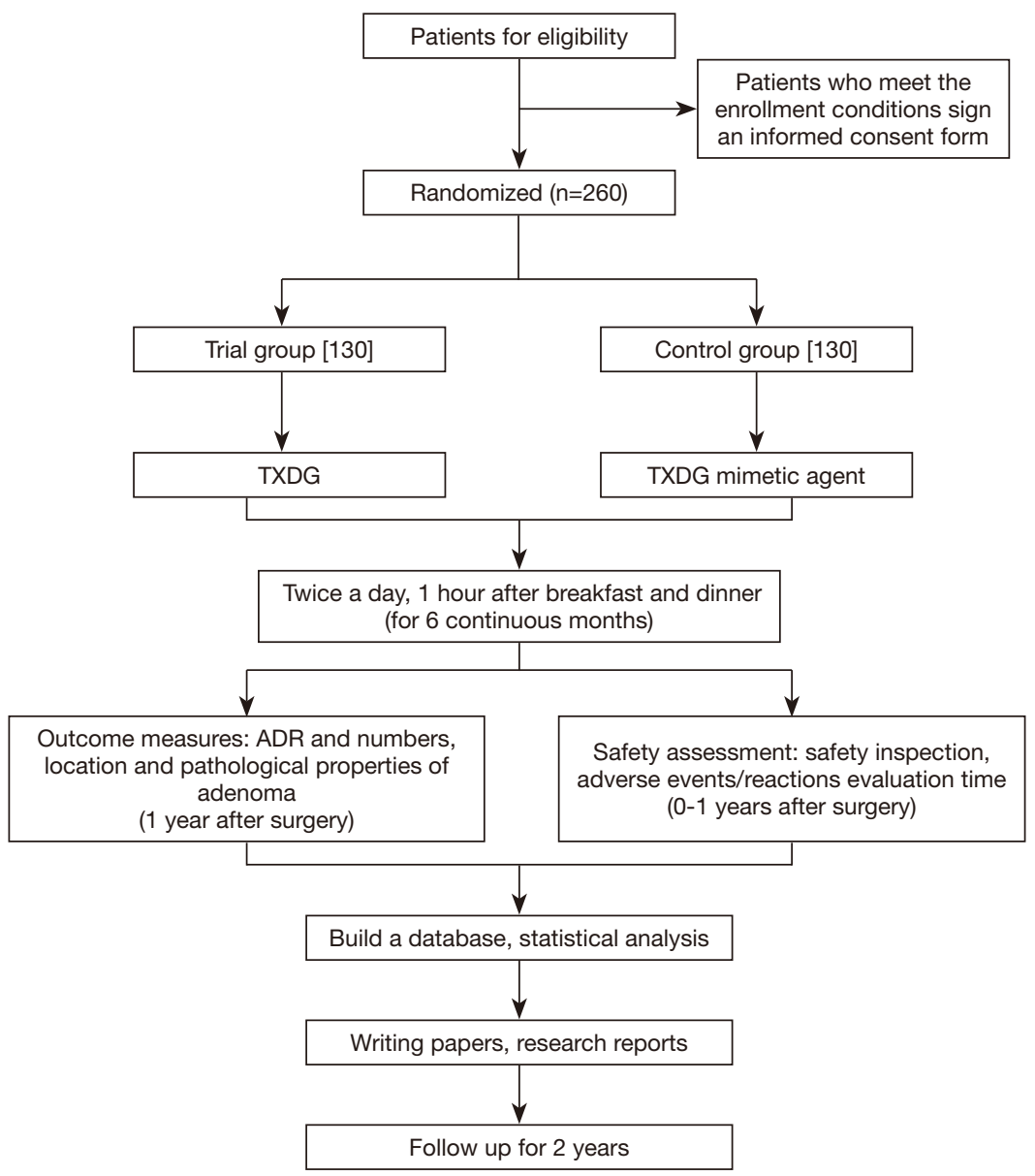

Figure 1 A flowchart showing the process of the proposed study.

intervention was $12.5 \%$, compared to a recurrence rate of $30.6 \%$ in patients who underwent colonoscopy treatment. In line with the 1:1 parallel control principle, for a doublesided significance level of 0.05 and a power of $90 \%(\alpha=0.05$, $\beta=0.1$ ), the PASS statistical analysis software package was used to calculate the sample size. Moreover, considering a $20 \%$ loss to follow-up, the sample size was determined to be 260 cases ( $n=130$ in each group).

\section{Randomization}

The methods of central stratification and block randomization will be used in all 5 hospitals simultaneously. The unit group leader will generate a random arrangement for the treatment of 260 subjects with the aid of the SAS statistical analysis system. Each unit member enters the basic information of the cases into the system and the eligible cases will be automatically classified into the experimental group or the control group for corresponding processing.

\section{Blinding}

This trial is a blinded trial, divided into the TXDG group and the TXDG mimetic agent group. The double levels of blinding are sealed separately and handed over to the leaders and sponsors of the clinical research. Each hospital will receive an emergency letter, as well as the trial drugs, which will be stored properly until the conclusion of the trial. The patients and investigators (including statisticians) will be blinded to the treatments. In the event of a serious adverse event or when further intervention is required and information regarding the actual medication administered is needed, the treatment can be unblinded with the permission of the person in charge of the research center, and a report should be submitted to the trial team leader within 
24 hours.

\section{Participants}

Patients will be enrolled in the study if they meet the following inclusion criteria:

(I) Participants aged 40-70 years, regardless of gender;

(II) The participant is diagnosed with pathologically confirmed CRAs (including tubular adenoma, tubulovillous adenoma, and villous adenoma), and the number of adenomas is more than 1 but no more than 6 ;

(III) All CRAs have undergone endoscopic treatment and no residual adenomas are detectable, within 1 month after the procedure;

(IV) Participants have provided informed consent.

The following exclusion criteria were applied:

(I) Participants complicated with colorectal malignancies (including early cancer and invasive cancer), or patients with a history of colorectal malignancies;

(II) Participants with a medical history of inflammatory bowel disease such as ulcerative colitis and Crohn's disease;

(III) Participants who are pregnant, breastfeeding, or expecting pregnancy;

(IV) Participants presenting with severe liver, kidney, heart, brain, or lung dysfunction and cannot tolerate colonoscopy treatment and clinical intervention;

(V) Participants with drug allergies and allergies to other components used in the trial;

(VI) Participants suffering from severe mental illness;

(VII) Participants with a history of alcohol or drug abuse;

(VIII) Participants with frequent changes in their working environment or other situations and are thus easily loss to follow-up;

(IX) Participants who are regularly taking nonsteroidal anti-inflammatory drugs (NSAIDs), calcium, or vitamin D supplementation;

(X) Participants who have been involved in other clinical trials within the past 3 months.

\section{Trial medicine}

Trial medicines are TXDG and TXDG simulants, both provided by Guangdong Yifang Pharmaceutical Co. Ltd. The shape, size, taste, color, package, and lot number of the TXDG simulants are no different from that of the TXDG.

\section{Intervening measures}

\section{Basic treatment}

Participants will be given health education, which includes explaining the related knowledge of CRA and providing the patients with guidance for reasonable diet (increase of dietary fiber intake (6), reduction of red meat intake), temperance of smoking and alcohol, regular exercise, body weight control (7), and emotion management.

\section{The TXDG group}

Participants will take a bag of TXDG with warm water twice a day, 1 hour after breakfast and 1 hour after dinner for 6 continuous months (28 days per month). Subjects will be contacted by telephone every month and monitored for adherence, illnesses, other medications, as well as side effects. Additionally, participants will be required to attend 4 follow-up visits, and the evaluation requirements during these follow-up sessions are shown in Table 2.

\section{TXDG mimetic agent group}

The control group will be administered the TXDG mimetic and followed-up in accordance with the experimental group.

\section{Colonoscopy procedures}

\section{The qualifications of the endoscopist}

The endoscopist will meet the following criteria:

(I) Proficiency in colonoscopy and magnifying staining endoscopy, with more than 3 years of working experience as a deputy chief physician;

(II) Has diagnosed and treated more than 2,000 cases by colonoscopy; and

(III) Has a detection rate of adenoma in patients with first colonoscopy of more than $15 \%$.

\section{Colonoscopy quality control}

The colonoscopy must meet the following quality control criteria: the Boston Bowel Preparation Score (BBPS) (8) should be above 6 points; the cecal scope-forward rate should be $95 \%$; and the withdrawal time should be more than 
Table 2 Trial schedule for participants

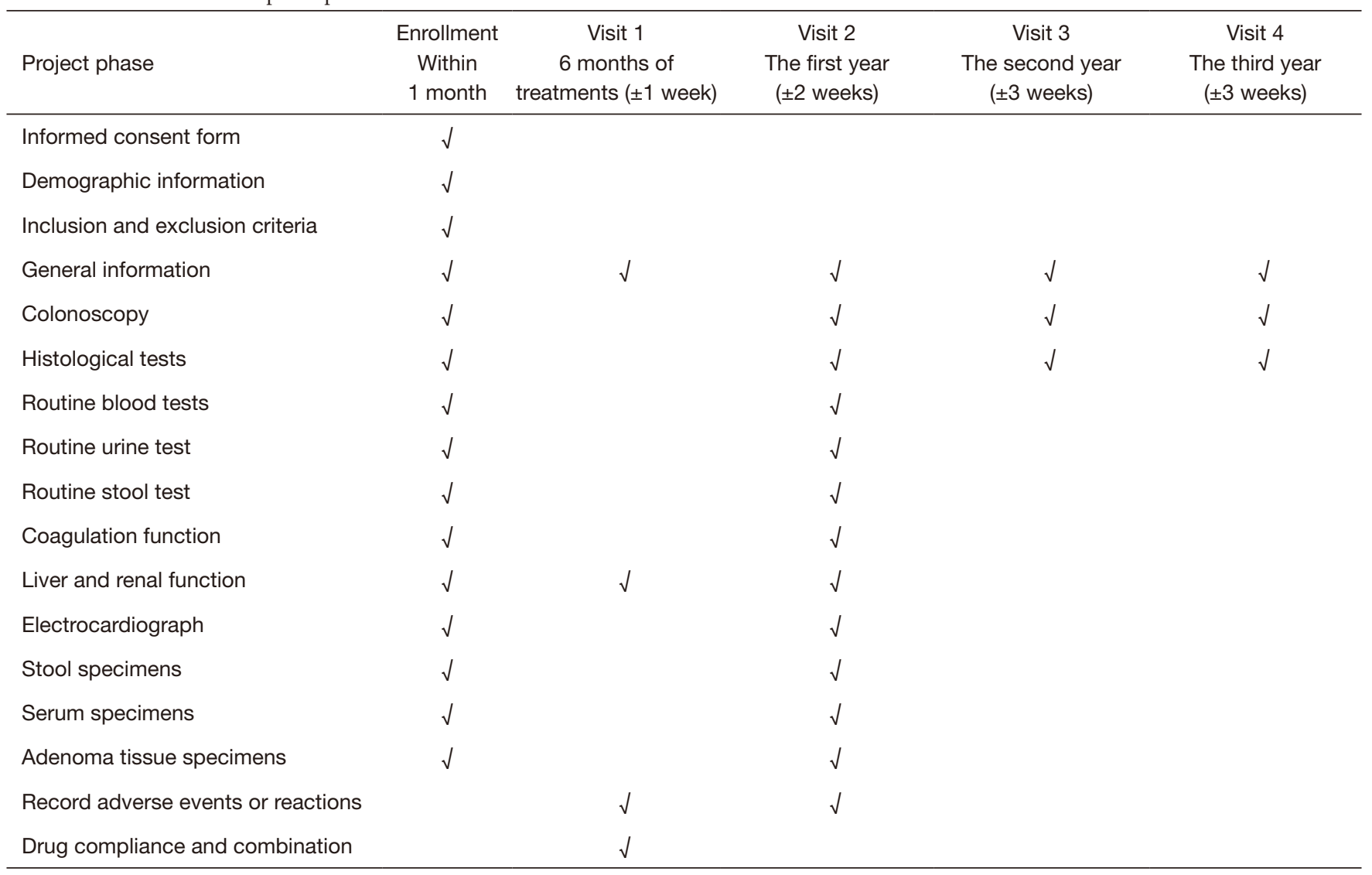

6 minutes, which improves the detection rate of adenomas. To reduce the rate of missed diagnosis, enteroscopy was performed by tandem colonoscopy (two scope-forward enteroscopes were performed in the same patient).

\section{Agent quality control}

The TXDG mimetic agent is prepared by a workshop pharmaceutical company that meets the requirements of Good Manufacturing Practice (GMP) and has passed quality inspections.

\section{Establishment and training of the operating specifications}

The standard operating procedures (SOPs) has been compiled by the research team, including procedures for colonoscopy, procedures for bowel preparation, procedures for endoscopic treatment of CRA, procedures for handling specimens after endoscopy, etc., and unified training will be provided to improve consistency.
Independent third-party observations and evaluation Efficacy evaluation and follow-up data will be observed and recorded by independent third-party researchers.

\section{Outcome measures}

\section{The primary outcome measure}

The primary outcome measure is the adenoma detection rate (ADR) which is the proportion of patients in each group with one or more CRAs detected during the colonoscopy re-examination.

\section{The secondary outcome measures}

The secondary outcome measures will assess the numbers, location, and pathological properties of the adenomas, as well as the polyp detection rate. The number of adenomas is the average number of recurrent CRAs per patient in each group. The location of the recurring colorectal adenoma (CRA) will be determined (left colon or right colon). 
The pathological properties of the recurring adenomas will be classified according to tubular adenomas, villous adenomas, villous tubular mixed adenomas, or neoplasia (no intraepithelial neoplasia, uncertain intraepithelial neoplasia, low grade intraepithelial neoplasia, or highgrade intraepithelial neoplasia). The polyp detection rate is defined as the proportion of patients in each group with one or more colorectal polyps during the colonoscopy reexamination.

\section{Statistical analysis}

The demographic characteristics of the treatment group and the control group will be analyzed to investigate the balance and comparability of the two groups. The effectiveness and safety indexes of the two groups will be compared.

Difference in quantitative data such as age, blood pressure, and heart rate will be compared between the experimental and control groups using the t-test for comparison of two samples, and the rank sum test for nonnormal distribution or unevenness of variance. For the comparison of two categorical indices, the Chi-square test or the exact probability method will be used. All statistical tests are two-sided, and a $\mathrm{P}$ value less than or equal to 0.05 is considered statistically significant. Unconditional stepwise logistic regression analysis is used for the analysis of factors affecting recurrence. Statistical analyses will be completed by unblinding.

\section{Safety assessment}

Laboratory indicators including routine blood tests, routine urine tests, routine stool tests, occult blood tests, coagulation tests, electrocardiogram (at 0 and 12 months), liver function tests, and kidney function tests (at 0,6 , and 12 months) will be performed. Adverse events will be monitored during the first year.

\section{Data management}

\section{Research medical records}

A special "study record" is designed for this study to completely preserve the primary data of this clinical research because in China, most of the outpatient medical records are kept by patients themselves. The study record for each patient, which is composed of the outpatient medical records and the inpatient medical records, is the original document and should be kept in the hospital.

\section{Data recording}

The following requirements for the study records should be adhered to:

(I) The study record must ensure that the data documented are timely, complete, accurate, and true;

(II) The study record can only be corrected by underlining the incorrect information and noting the revised data, along with the signature and date of the researcher. Data shall not be erased or written over on the original record;

(III) The laboratory test results of outpatients and inpatients must be documented in the "Physical and Chemical Test Results Report Form" in the study medical record.

After completing the observation procedures for a participant, the researcher should submit the "Research Medical Record", "Informed Consent Form”, and the "Patient Medication Record Card" to the project manager of the unit for examination, signature, and preservation within 3 working days. The data will then be transferred onto Case Report Forms (CRFs). Each center will arrange specialized electronic CRF input personnel. After completing each subject observation, the researcher will submit the trial data to the CRF input personnel who must review the study notes to ensure it is complete and meets the timeframe requirements.

\section{Data auditing}

Data auditing will be conducted by inspectors who will frequently visit the research centers in accordance with the quality control requirements of clinical research. The inspector examines each research medical record and fills in the "Inspector Audit Page" one by one. The consistency between the source data and the electronic CRF data shall be checked by two inspectors and the Data Consistency Check Report shall be completed. If an error is detected when filling in the data, researchers should submit the Data Consistency Check Error Correction Report and the error should be corrected by the electronic CRF data administrator in accordance with the report.

\section{Data inspection and blind review}

Prior to database establishment, the data must be inspected, and a blind review conducted. If there are errors in the number range and logic check, they will be filed in the problem list (query list), and the researcher must rectify the queries on the list. The list of questions 
should be stored securely. After completing the Data Inspection Report and the Data Blind Audit Report, the database will be locked. Relevant documents for quality control should be kept, such as the original records of data consistency check, numerical range and logic check, the original records of blind audits, and the records of questions regarding communications between researchers and inspectors.

\section{Adverse events}

All adverse events such as gastrointestinal reactions, liver damage, and renal failure will be recorded and graded in detail throughout the study. In the event of a serious adverse reaction, the researcher will provide all necessary treatment and report to the ethics committee of the Guangdong Provincial Hospital of Traditional Chinese Medicine.

\section{Discussion}

The high recurrence rate of colorectal adenomatous polyps after resection is a major hinderance in the prevention and treatment of CRC. However, to date, there is no exact and effective chemotherapeutic treatment. Previous randomized trials have failed to detect an overall efficacy of calcium or vitamin D3 supplementation against CRA recurrence (9). Other randomized trials have shown that aspirin and cyclooxygenase $2(\mathrm{COX}-2)$ inhibitors play a role in reducing the risk of CRA recurrence in patients with a history of CRC or CRAs. Nevertheless, COX2 inhibitors may cause gastrointestinal damage and are associated with serious cardiovascular events. In addition, the efficacy of these agents in reducing the recurrence of CRAs does not persist and may in fact increase the risk of adenoma recurrence following withdrawal (10). TCM has its own unique understanding of the occurrence of colorectal adenomatous polyps. Recent evidence has shown that TCM is safe and has few side effects when used to prevent the recurrence of CRAs. However, there is a lack of high-quality large scale randomized controlled trials to support this. Additionally, it is generally inconvenient to decoct TCM and difficult to consume TCM decoctions. This, together with the lack of any effective patents on Chinese medicine, has limited the popularization and application of TCM.

The TXD is a TCM decoction synthesized by Professor Yunjian Luo. It has been used for more than a decade in the Department of Gastroenterology, Guangdong Provincial
Hospital of TCM, with remarkable preventative effects on the recurrence of CRAs. According to Professor Luo, the weakness of the spleen and stomach, the endogenous damp turbidity, and the stagnation of blood stasis, which together cause the disease, are the important pathological basis for the recurrence of CRAs. The evolution of CRC is closely related to the imbalance of various intra-cellular signal transduction cascades, including protein kinase B (AKT), mitogen-activated protein kinase 1 (MAPK), and signal transducer and activator of transcription 3 (STAT3), as well as crosstalk between these signaling networks (11). Our previous research suggested that TXD may help decreasing the recurrence rate of colorectal adenoma after operation, possibly by inhibiting autophagy, decreasing abnormal cell proliferation, and relieving inflammation of the colonic mucosa (12). Much research have shown that the different components of TXD have anti-tumor effects. In this formula, Coix lacrymajobi L.Vra.mayuen (Roman) Stapf (Yiyiren) is the sovereign medicinal in TXD. According to Chinese medical literature, clinical trials have demonstrated that the Coix extract is beneficial for the treatment of cancers including lung cancer, breast cancer, and hepatocellular carcinoma. Laboratory studies on cancer cell lines have suggested that the mechanisms of action may involve inhibiting cell replication or inducing apoptosis (13). Codonopsis pilosula (Franch.)Nannf. (Dangsheng) and Atractylodes macrocephala koidz (Baizu) are the Chen (minister) components, which synergize with the Jun component to strengthen its therapeutic effects. Radix Codonopsis (Dangshen), recorded in Sheng Nong's herbal classic, is often used in formulas to treat patients with a deficiency in spleen qi (symptoms of spleen deficiency include anorexia, fatigue, as well as abdominal distension) and tumors (14). Atractylodes macrocephala koidz (Baizu) can improve gastrointestinal function and inhibit tumor progression. The molecular mechanisms of tumor progression suppression is partially mediated via resolution of the inflammatory environment (15). The Zuo (assistant) component Hedyotis diffusa Willd. plays an important role in treating malignant tumors since its extracts possess evident anti-tumor activities (16).

This randomized, controlled, double-blinded, and multicenter trial will further demonstrate the potential of TXDG as an adjuvant treatment in preventing the recurrence of CRAs. This, together with an understanding of its underlying mechanisms will provide direct evidencebased support for the use of TCM in the prevention and treatment of colorectal adenomatous polyps. 


\section{Trial situation}

At the time of submission of this manuscript, participant recruitment for the trial is in progress. The trial will be conducted from December 2020 to December 2022. A total of 260 patients will be recruited in this trial. This is protocol version number 2 and the date is August 7, 2020.

\section{Acknowledgments}

Funding: Special funding for clinical research of Guangdong Provincial Hospital of Traditional Chinese Medicine (No. YN10101914).

\section{Footnote}

Reporting Checklist: The authors have completed the SPIRIT reporting checklist. Available at http://dx.doi.org/10.21037/ apm-21-650

Conflicts of Interest: All authors have completed the ICMJE uniform disclosure form (available at http://dx.doi. org/10.21037/apm-21-650). The authors have no conflicts of interest to declare.

Ethical Statement: The authors are accountable for all aspects of the work in ensuring that questions related to the accuracy or integrity of any part of the work are appropriately investigated and resolved. The trial complies with the Good Clinical Practice Guidelines and the Declaration of Helsinki (as revised in 2013). Ethics approval of this trial has been obtained from the ethic committee of the Guangdong Provincial Hospital of Traditional Chinese Medicine (No. BF2020-103-01). All subjects will be requested to sign written informed consent forms.

Open Access Statement: This is an Open Access article distributed in accordance with the Creative Commons Attribution-NonCommercial-NoDerivs 4.0 International License (CC BY-NC-ND 4.0), which permits the noncommercial replication and distribution of the article with the strict proviso that no changes or edits are made and the original work is properly cited (including links to both the formal publication through the relevant DOI and the license). See: https://creativecommons.org/licenses/by-nc$\mathrm{nd} / 4.0 /$.

\section{References}

1. Sung H, Ferlay J, Siegel RL, et al. Global cancer statistics 2020: GLOBOCAN estimates of incidence and mortality worldwide for 36 cancers in 185 countries. CA Cancer J Clin 2021.

2. Cotton S, Sharp L, Little J. The adenoma-carcinoma sequence and prospects for the prevention of colorectal neoplasia. Crit Rev Oncog 1996;7:293-342.

3. Shi $X$, Yang $Z, W u$ Q, et al. Colorectal adenoma recurrence rates among post-polypectomy patients in the placebo-controlled groups of randomized clinical trials: a meta-analysis. Oncotarget 2017;8:62371-81.

4. Vijayananthan A, Nawawi O. The importance of Good Clinical Practice guidelines and its role in clinical trials. Biomed Imaging Interv J 2008;4:e5.

5. Zhang BP, Zhao XY, Liu G, et al. TCM Intervention of Adenomatous Colorectal Polyps Based on Theory "Preventive Treatment of Diseases". Chinese General Practice 2012;15:2718-20.

6. Kunzmann AT, Coleman HG, Huang WY, et al. Fruit and vegetable intakes and risk of colorectal cancer and incident and recurrent adenomas in the PLCO cancer screening trial. Int J Cancer 2016;138:1851-61.

7. Bardou M, Barkun AN, Martel M. Obesity and colorectal cancer. Gut 2013;62:933-47.

8. Calderwood AH, Jacobson BC. Comprehensive validation of the Boston Bowel Preparation Scale. Gastrointest Endosc 2010;72:686-92.

9. Calderwood AH, Baron JA, Mott LA, et al. No Evidence for Posttreatment Effects of Vitamin D and Calcium Supplementation on Risk of Colorectal Adenomas in a Randomized Trial. Cancer Prev Res (Phila) 2019;12:295-304.

10. Veettil SK, Lim KG, Ching SM, et al. Effects of aspirin and non-aspirin nonsteroidal anti-inflammatory drugs on the incidence of recurrent colorectal adenomas: a systematic review with meta-analysis and trial sequential analysis of randomized clinical trials. BMC Cancer 2017;17:763.

11. Feng J, Jin Y, Peng J, et al. Hedyotis diffusa willd extract suppresses colorectal cancer growth through multiple cellular pathways. Oncol Lett 2017;14:8197-205.

12. Li Y, Zhong CL, Yang SP, et al. Effect of Tiaochang Xiaoliu Formula on the Recurrence Rate of Patients with Colorectal Adenoma and the Expression of Beclin1, p53 and Cox-2 in Colon Tissue. Journal of Traditional Chinese 
Medicine 2021;62:424-7.

13. Woo JH, Li D, Wilsbach K, et al. Coix seed extract, a commonly used treatment for cancer in China, inhibits NFkappaB and protein kinase C signaling. Cancer Biol Ther 2007;6:2005-11.

14. Zhou Q, Chen YG, Xiao J, et al. Traditional Chinese medicine (Xiaoai Jiedu Decoction) as an adjuvant treatment for prevention new colorectal adenomatous polyp occurrence in post-polypectomy: Study protocol for a randomized controlled trial. Medicine (Baltimore) 2019;98:e16680.

15. Wan G, Xie M, Zhang X, et al. Chang-wei-qing, a Chinese herbal formula, ameliorates colitis-associated tumour

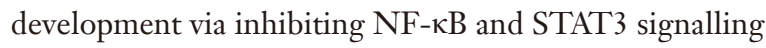
pathway. Pharm Biol 2019;57:231-7.

16. Wang C, Zhou X, Wang Y, et al. The Antitumor Constituents from Hedyotis Diffusa Willd. Molecules 2017;22:2101.

(English Language Editor: J. Teoh)

Cite this article as: Zhong C, Wu S, Chen C, Huang H, Mo J, Yang X, Chen Y, Zhang B. Tiaochang Xiaoliu Decoction Granules prevent the recurrence of colorectal adenoma: a study protocol for a randomized controlled trial. Ann Palliat Med 2021;10(4):4897-4905. doi: 10.21037/apm-21-650 